\title{
Extracardiac Anastomosis in Coronary Ostial Occlusion
}

\author{
N. G. SANERKIN \\ From Group Pathology Laboratory, St. David's Hospital, Cardiff
}

In his review of the physiology of the coronary circulation, Wiggers (1936) enumerated three types of compensatory anastomosis that may develop after coronary occlusion: (1) through development of new intercoronary communications, (2) through formation of extracardiac communications, and (3) through enlargement of arterio-luminal channels. Widespread minor anastomoses are known to exist between branches of the coronary arteries and branches of extracardiac arteries around the major vessels at the root of the heart, and it is believed (Hudson, Moritz, and Wearn, 1932) that this potential extracardiac coronary collateral circulation may be of significance in compensating for sclerosis of the large trunks of the coronary arteries. It is not generally appreciated, however, that such extracardiac collateral arteries may attain a considerable calibre, as in the case reported here.

\section{Case Report}

A 70-year-old man was "permanently maintained" at a hospital for war pensioners. He was in receipt of a 100 per cent disability pension in respect of "arthritis of the hips", and had had bilateral subtrochanteric osteotomies of the left femur in 1947 and of the right femur in 1949. A diagnosis of ankylosing spondylitis had been made, affecting mainly the sacro-iliac joints with complete bony ankylosis, involving the spine to.a lesser degree. Since 1958 he had been in low-grade congestive cardiac failure. There was slight cardiomegaly. On some occasions a soft systolic murmur was heard at the apex, but there is no record of an aortic incompetence murmur. Electrocardiogram showed evidence of left ventricular hypertrophy and ischaemia with left bundlebranch block. Radiologically there was aortic unfolding with considerable calcification in the knuckle. Serum Wassermann reaction, Price's precipitation reaction, and gonococcal complement-fixation test were negative. He had been known to have a chronic duodenal ulcer for many years, with radiological evidence of gross pyloric and duodenal deformity. During the summer months of 1966 he had recurrent episodes of haematemesis and melaena, but after full investigation in
September 1966 it was decided that an operation was not indicated. He died early in October 1966.

At necropsy, there was a huge chronic peptic ulcer in the posterior wall of the duodenum just distal to the pyloric sphincter, eroding deeply into the pancreas. The immediate cause of death was massive pulmonary embolism secondary to leg vein thrombosis. Among the incidental findings was a chronic aortitis which, in view of the negative Wassermann reaction and the existence of ankylosing spondylitis, was presumed to be an example of spondylitic aortitis, an account of which condition can be found in Hudson (1965). The aorta was considerably dilated and scarred in its thoracic portion. Intimal scarring was particularly prominent in the proximal part of the ascending aorta, with much ulceration and calcification, maximal above the left coronary cusp of the aortic valve and extending onto the wall of the left coronary sinus of Valsalva, where it caused pinpoint stenosis of the left coronary ostium (Fig. 1). Severe scarring was present between the coronary cusps of the aortic valve, with appreciable widening of the commissure. There was some fibrosis on the walls of the other sinuses of Valsalva, but the two ostia of the right coronary artery and the other commissures were unaffected. The main trunk of the left coronary artery appeared as a solid fibrous cord, due to old organized thrombotic occlusion of this segment, the thrombosis having presumably followed from occlusion of the ostium. The right coronary artery and the left coronary artery beyond the occluded main trunk were widely patent and free from significant atheroma. Immediately beyond the occluded segment, the anterior descending branch of the left coronary artery was thin-walled and aneurysmally distended, and was joined by three anastomotic arteries. Two of these were intercoronary anastomoses (Fig. 2) arising from the right coronary artery (the upper about $0.2 \mathrm{~cm}$. diameter on cross-section, the other of somewhat smaller calibre). The third collateral artery (about $0.2 \mathrm{~cm}$. on cross-section) coursed downwards and to the left across the anterior aspect of the pulmonary trunk (Fig. 2 and 3), proceeded along a tortuous course in the epicardial fat, and entered the left coronary artery from behind. This particular artery was not identified until after completion of the necropsy, so that its exact extracardiac connexions could not be determined, but it is reasonable to assume that it joined 


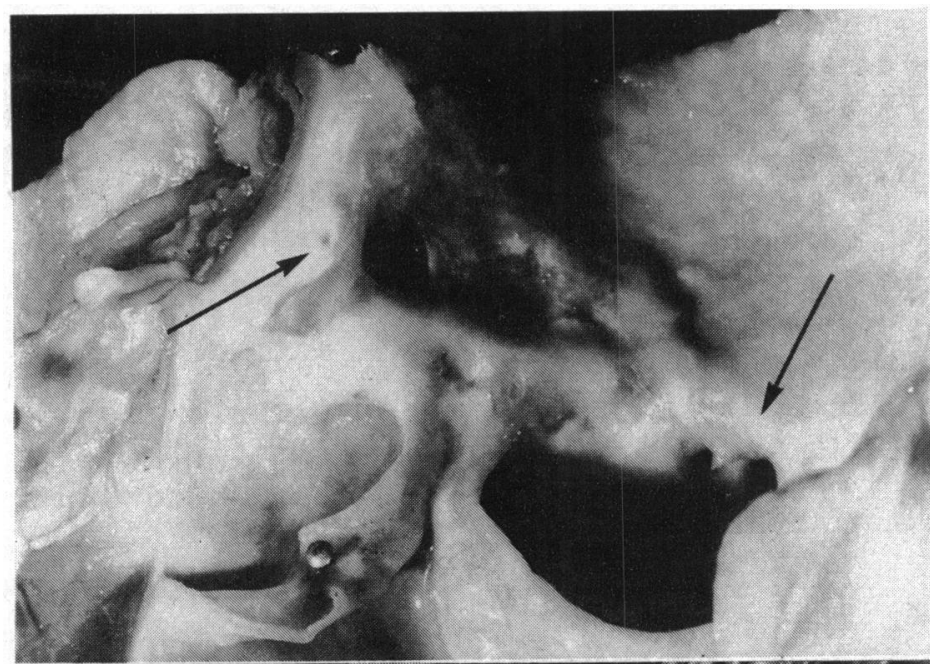

Fig. 1.-Coronary cusps of aortic valve. Dense fibrous tissue on wall of left coronary sinus causing pin-point stenosis of left coronary ostium (arrow on left). Commissure scarred and widened. Right coronary ostia patent (arrow on right). Dark patch above left coronary ostium is due to ulcerating atheroma.

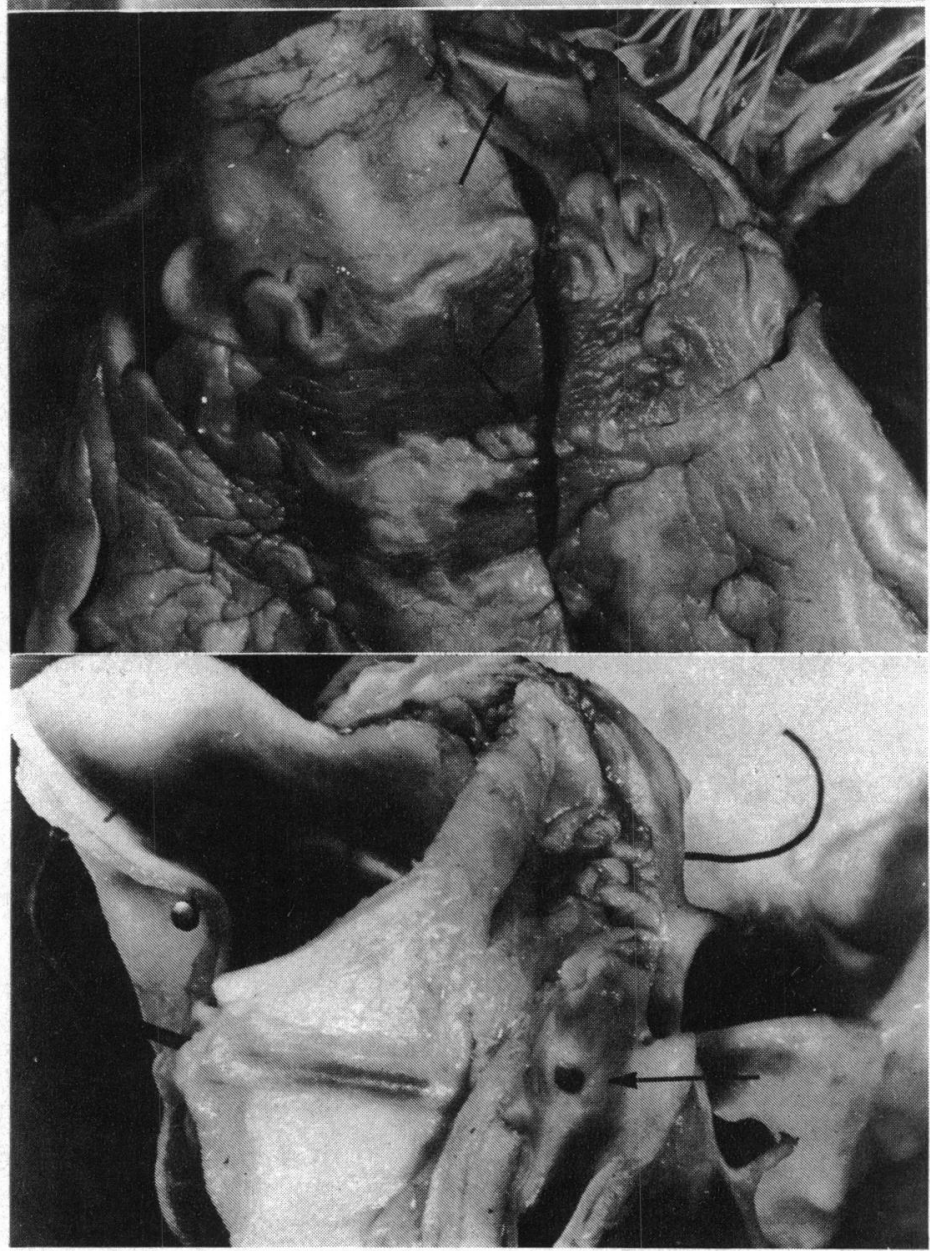

Fig. 2.-External surface of heart over conus of right ventricle, showing (bottom arrows) intercoronary and (top arrow) extracardiac anastomoses.

Fig. 3.-Anterior aspect of pulmonary trunk showing extracardiac collateral artery. Arrow points to entry of this vessel into the anterior descending coronary artery. 
one of the numerous arteries supplying the mediastinum. The heart ( 365 g.) was slightly dilated but not significantly hypertrophied. Fairly localized irregular fibrosis was found in the left ventricular myocardium at the apex. There was no evidence of focal endocardial fibrosis on the left ventricular septum below the aortic valve, such as is often found in aortic incompetence and usually described as the "jet lesion" of the latter.

\section{Discussion}

In occlusive disease of the coronary arteries, the anatomical distribution and functional efficiency of collateral anastomoses depend on various factors, such as the site and extent of the occlusion, the rate at which it develops, and the condition of the arterial tree beyond the occlusion. The combination of factors most favourable to the development of substantial coronary anastomoses is probably seen in chronic aortitis, syphilitic or otherwise, in which the occlusion affects the ostia, is a gradual and insidious process, and the arteries beyond the occlusion often remain free from significant atheroma. The ostial stenosis leads to a lowering of the coronary diastolic pressure (Wiggers, 1936) which helps reverse the flow in the peripheral branches of the affected coronary tree, initiating collateral flow into the latter. Where only one coronary ostium is affected, as in the present case, both intercoronary and extracardiac anastomoses are likely to develop.

Where both coronary ostia are affected, intercoronary anastomosis is discouraged and extracardiac anastomoses become correspondingly more important. Cases of complete occlusion of both coronary ostia have been reported by Leary and Wearn (1930), who ascribed the ability of such patients to live and work to the belief that the Thebesian veins supplied the compensatory circulation necessary for the functioning of the heart muscle. The present case demonstrates, however, that extracardiac anastomoses of considerable calibre are possible even in single coronary ostial occlusion with well-developed intercoronary anastomoses, when the latter would tend to prevent full development of extracardiac collaterals. The Thebesian system (Wiggers, 1936) is potentially the least efficient of the possible collaterals and its role in complete coronary ostial occlusion must remain debatable.

In coronary atheroma, as in syphilitic or spondylitic coronary ostial occlusion, stenosis tends to promote the development of collaterals (Ravin and Geever, 1946; Zoll, Wessler, and Schlesinger, 1951). However, progressive atheroma hinders their full development and maintained efficiency. Any collaterals, whether intercoronary or extracardiac, suffer eventual atheromatous involvement with loss of their compensatory value (Anitschkow et al., 1964).

\section{Summary}

A case is described of chronic aortitis, presumably spondylitic, with occlusion of the left coronary ostium and consequent development of significant extracardiac as well as intercoronary anastomoses.

I am indebted to Mr. W. T. Adams, H.M. Coroner for the City of Cardiff, Mr. Dillwyn Evans, Consultant Orthopaedic Surgeon, and Dr. C. G. Bryan, Medical Superintendent, Rookwood Hospital, for their consent to the publication of this case. My thanks are also due to Mr. S. W. Midgley for the photographs.

\section{References}

Anitschkow, N. N., Wolkoff, K. G., Kikaion, E. E., and Pozharisski, K. M. (1964). Compensatory adjustments in the structure of coronary arteries of the heart with stenotic atherosclerosis. Circulation, 29, 447.

Hudson, C. L., Moritz, A. R., and Wearn, J. T. (1932). The extracardiac anastomoses of the coronary arteries. f. exp. Med., 56, 919.

Hudson, R. E. B. (1965). Cardiovascular Pathology, pp. 1088-1092. Arnold, London.

Leary, T., and Wearn, J. T. (1930). Two cases of complete occlusion of both coronary orifices. Amer. Heart F., 5 , 412.

Ravin, A., and Geever, E. F. (1946). Coronary arteriosclerosis, coronary anastomoses and myocardial infarction. A clinicopathologic study based on an injection method. Arch. intern. Med., 78, 125.

Wiggers, C. J. (1936). The physiology of the coronary circulation. In Diseases of the Coronary Arteries and Cardiac Pain, pp. 57-108. Ed. by R. L. Levy. Macmillan, New York.

Zoll, P. M., Wessler, S., and Schlesinger, M. J. (1951). Interarterial coronary anastomoses in the human heart, with particular reference to anemia and relative cardiac anoxia. Circulation, 4, 797. 